\title{
Tolerance averaging of heat conduction in transversally graded laminates
}

\author{
Jarosław Jędrysiak • Alina Radzikowska
}

Received: 10 July 2010 / Accepted: 28 December 2010 / Published online: 15 January 2011

(C) The Author(s) 2011. This article is published with open access at Springerlink.com

\begin{abstract}
It is considered a heat conduction in a layer made of two conductors distributed in the form of laminas with varied thicknesses. Macroscopic (averaged) properties of the layer are continuously "transversally" graded across its thickness (TGL layer), cf. Fig. 1. The aim of the paper is to present and apply an averaged model of the heat conduction, obtained within the tolerance averaging technique, discussed in the book edited by Woźniak et al. (Thermomechanics of microheterogeneous solids and structures. Tolerance averaging approach, Łódź, Wydawnictwo Politechniki Łódzkiej, 2008). It is shown that the proposed model describes the microstructural effect on the heat conduction of the TGL layer. Moreover, results obtained within this model are compared to results by the higher order theory (cf. Aboudi et al., Composites B, 30:777-832, 1999).
\end{abstract}

Keywords Functional composites · Transversally graded laminates $\cdot$ Laminate theory $\cdot$ Heat conduction $\cdot$ Effect of the microstructure

J. Jędrysiak ( $\varangle) \cdot$ A. Radzikowska

Department of Structural Mechanics, Technical University

of Łódź, al. Politechniki 6, 90-924 Łódź, Poland

e-mail: jarek@p.lodz.pl

A. Radzikowska

e-mail: alina.radzikowska@p.lodz.pl

\section{Introduction}

In this paper a laminated layer is the main object of considerations. This layer is made of two conductors, non-periodically distributed as micro-laminas along the layer thickness. The laminated composite is assumed to have macroscopic properties, which continuously vary across its thickness. Similar materials are called functionally graded materials (FGM), cf. Suresh and Mortensen [27]. A fragment of the layer is shown in Fig. 1. In Fig. 1a there is a layer in a macroscale, and in Fig. 1b-in a micro-scale. Laminates of this kind will be called transversally graded laminates (TGL).

Because the exact description of the geometry of the microstructure of FGM-type composites is usually impossible, thermomechanical phenomena in these composites can be investigated only in the framework of micromechanical models with idealised geometries. Assumptions of idealisation can be similar to those applied to describe composites, which are macroscopically homogeneous. Although functionally graded materials are not homogeneous in a macro-scale, their overall behaviour can be analysed by adopted and modified methods, which are used for macroscopically homogeneous materials, e.g. periodic composites (laminates). Certain averaged methods, applied to determine properties of FGM-type composites, are discussed in the paper Reiter, Dvorak and Tvergaard [21] and in the monograph by Suresh and Mortensen [27]. It is necessary to mention these methods which are 

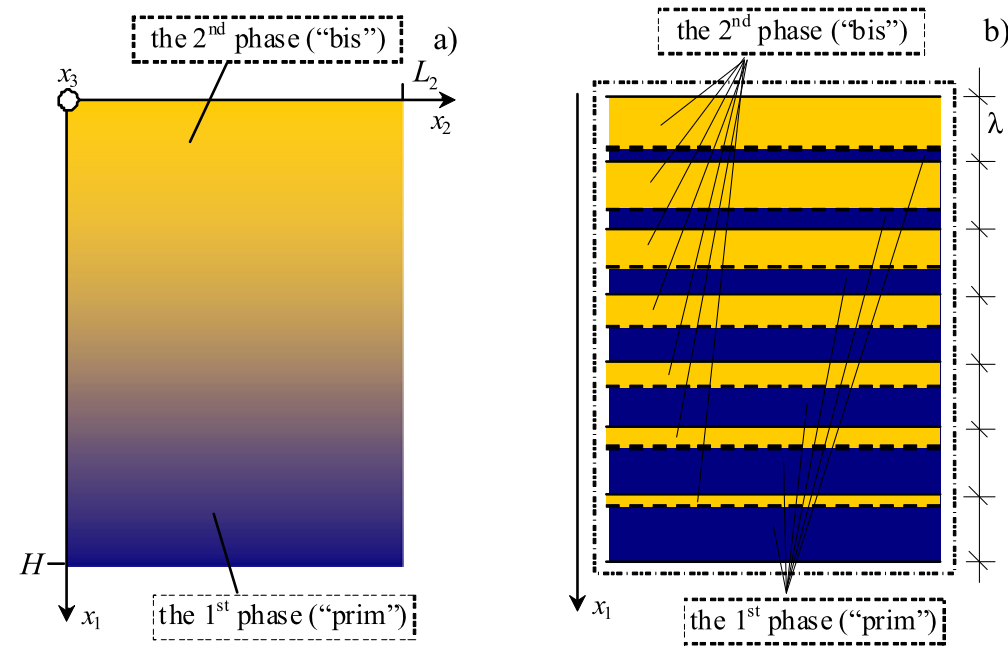

Fig. 1 A fragment of a transversally graded laminated layer in: (a) a macro-scale, (b) a micro-scale

based on the asymptotic homogenisation, cf. Jikov, Kozlov and Oleinik [12], which are applied to heat conduction problems in periodic structures. Various thermoelastic problems of laminates are also investigated using models with microlocal parameters, e.g. a heat conduction in periodic composites is presented in Matysiak [17]. Unfortunately, the governing equations of the aforementioned models do not take into account the effect of microstructure size on the overall behaviour of periodic laminates. In the framework of the homogenisation approach problems of heat conduction and thermal stresses for functionally graded composites are analysed, e.g. by Itoh, Takahashi and Takano [7]. Some theoretical and numerical results of thermomechanical problems of functionally graded structures are shown in a lot number of papers. Here, it can be mentioned some of them. In the paper by Kantor, Smetankina and Shupikov [13] orthonormal Legendre polynomials are applied to nonstationary heat conduction in laminated elements. Stochastic thermal stresses in an FGM annular disc are analysed by Chiba [3]. A Green's function approach is proposed to analyse heat conduction and thermoelasticity problems by Kim and Noda $[15,16]$. A collocation method with higher-order plate theories are applied to analyse vibrations of functionally graded plates by Roque, Ferreira and Jorge [22] and Ferreira, Batra, Roque, Qian and Jorge [4]. Some analytical solutions to thermoelastic problems for a thick strip and hollow cylinder are obtained by using the method of Laplace transformation in papers Ootao and Tani- gawa $[19,20]$. Thermo-elastic-plastic properties of a FGM-type composite are predicted using a numerical homogenisation technique by Schmauder and Weber [25]. The boundary element method is used in a thermal analysis by Goldberg and Hopkins [5]. A certain meshless computational method is proposed by Sladek J., Sladek V. and Zhang [26]. A thermoelastic problem for cylindrical shells is analysed by a finite element model by Santos, Mota Soares C.M., Mota Soares C.A. and Reddy [24]. In the paper by Sadowski, Ataya and Nakonieczny [23] the FourierKirchhoff equation for the cylindrical plates is solved by using the finite difference method and the finite element method. Nonlinear vibrations of functionally graded beams are analysed using an analytical approach by Ke, Yang and Kitipornchai [14]. A dynamic behaviour of functionally graded shells is investigated by Tornabene and Viola [28]. An alternative modelling method for functionally graded materials, called the higher-order theory, is presented in Aboudi, Pindera and Arnold [1], and its reformulation by Bansal and Pindera [2].

The above drawback is omitted in averaged models based on the tolerance averaging technique, proposed for periodic composites by Woźniak Cz. and Wierzbicki [30]. In the framework of this method various problems of periodic structures were investigated, i.e. some problems of heat conduction for periodic composites were also analysed within this method, e.g. by Ignaczak and Baczyński [6], Woźniak M., Wierzbicki and Woźniak Cz. [31]; buckling of thin 
periodic plates by Jędrysiak [8]; wave propagation in multiperiodic composites by Jędrysiak and Woźniak Cz. [11]. Using this method equations with functional, periodic, highly-oscillating and also non-continuous coefficients can be replaced by the system of differential equations with constant coefficients, which describe the effect of the microstructure size on the overall behaviour of the periodic composite.

The tolerance averaging technique was also adopted to analyse various thermomechanical problems of FGM-type structures, e.g. a heat conduction in composites with transversal gradation by Jęrysiak and Radzikowska [10], or with longitudinally gradation by Michalak, Woźniak Cz. and Woźniak M. [18], stability problems for thin functionally graded plates by Jędrysiak and Michalak [9].

The overview of results concerning the tolerance averaging models for periodic and/or functionally graded composites and structures can be found in the monographs by Woźniak Cz. and Wierzbicki [30], Woźniak Cz., Michalak and Jędrysiak [29].

There are two aims of the paper. The first aim is to present certain averaged tolerance model of heat conduction in laminated layer with functionally graded macroscopic properties along its thickness. The second one is to apply this model to show some microstructural effects on the stationary heat conduction across laminas. Moreover, obtained results are compared with results calculated within the higher-order theory by Aboudi, Pindera and Arnold [1].

\section{Modelling foundations}

Let subscripts $i, j, \ldots$, run over $1,2,3$ and be related to the coordinate system $O x_{1} x_{2} x_{3}$; and also subscripts $\alpha, \beta, \ldots$, run over 2,3 , and be related to $O x_{2} x_{3}$. Introduce denotations: $\mathbf{x} \equiv\left(x_{2}, x_{3}\right), x \equiv x_{1} ; t$ for the time coordinate. Denote derivatives of $x_{i}$ by $\partial_{i}$. Let us also introduce denotations: $\nabla \equiv\left(\partial_{1}, \partial_{2}, \partial_{3}\right), \partial \equiv\left(\partial_{1}, 0,0\right)$, $\bar{\nabla} \equiv\left(0, \partial_{2}, \partial_{3}\right)$. Moreover, let $H$ be the layer thickness along the $x$-axis, and $L_{\alpha}$ be length dimensions along the $x_{\alpha}$-axes $(\alpha=2,3)$. Denoting by $\Omega \equiv(0, H)$, $\Xi \equiv\left(0, L_{2}\right) \times\left(0, L_{3}\right)$, the layer under consideration occupies the region $\Omega \times \Xi$ in the physical space. This layer is made of two materials distributed in $m$ laminas with the same thickness $\lambda(H=m \lambda)$. It is assumed that condition $\lambda \ll H$ is satisfied. Hence, thickness $\lambda$ can be called the microstructure parameter.
The "basic cell" in the interval $\Omega$ can be denoted by $\square \equiv(-\lambda / 2, \lambda / 2)$. Every $n^{\text {th }}$ lamina consists of two homogeneous sub-laminas with thicknesses $\lambda_{n}^{\prime}, \lambda_{n}^{\prime \prime}$, dependent on the argument $x$, cf. Fig. 1b. The sublaminas have properties described by: specific heats $c^{\prime}, c^{\prime \prime}$ and heat conduction tensors $\mathbf{K}^{\prime}, \mathbf{K}^{\prime \prime}$, with components $k_{i j}^{\prime}, k_{i j}^{\prime \prime}, i, j=1,2,3$. Denote material volume fractions in the $n^{\text {th }}$ lamina by $v_{n}^{\prime} \equiv \lambda_{n}^{\prime} / \lambda$, $v_{n}^{\prime \prime} \equiv \lambda_{n}^{\prime \prime} / \lambda$. Because it is assumed that sequence $\left\{v_{n}^{\prime}\right\}, n=1, \ldots, m$, is monotone and satisfies condition $\left|v_{n+1}^{\prime}-v_{n}^{\prime}\right| \ll 1$, for $n=1, \ldots, m-1$, the layer can be treated as made of a functionally graded material across laminas. Hence, this layer is called the transversally graded laminated layer (the TGL layer). Because $v_{n}^{\prime}+v_{n}^{\prime \prime}=1$ sequence $\left\{v_{n}^{\prime \prime}\right\}$ satisfies similar conditions. Thus, sequences $\left\{v_{n}^{\prime}\right\},\left\{v_{n}^{\prime \prime}\right\}, n=1, \ldots, m$, can be approximated by continuous functions $v^{\prime}(\cdot)$, $v^{\prime \prime}(\cdot)$. These functions describe the gradation of material properties along the $x$-axis and can be called the fraction ratios of materials. Let us introduce function $v(\cdot)$ defined by formula $v(\cdot) \equiv\left[v^{\prime}(\cdot) v^{\prime \prime}(\cdot)\right]^{\frac{1}{2}}$, called the non-homogeneity ratio. We assume that the above functions of fraction ratios are slowly-varying (cf. the book edited by Woźniak Cz., Michalak and Jędrysiak [29]).

Let us denote the unknown temperature field by $T$ and the thermal load (the intensity of heat sources) by $f$. Moreover, we assume that the heat conduction problem in the TGL layer can be analysed using the Fourier's model, i.e. this problem can be described by the equation:

$$
\nabla \cdot(\mathbf{K} \cdot \nabla T)-c \dot{T}=f
$$

for every instant $t$.

It can be observed that for the TGL layer all coefficients of (1), i.e. $\mathbf{K}=\mathbf{K}(x), c=c(x)$, are highlyoscillating, tolerance-periodic, non-continuous functions in $x$. Hence, this equation is not a good tool to investigate engineering problems. In order to obtain differential equations with continuous, smooth, slowly-varying functional coefficients instead of (1), the tolerance averaging technique will be applied, cf. the book by Woźniak Cz. and Wierzbicki [30], and the book edited by Woźniak Cz., Michalak and Jędrysiak [29]. 


\section{Tolerance averaging approach}

\subsection{Introductory concepts}

Let $f$ be an integrable function defined in $\bar{\Omega}$, which can also depend on $\mathbf{x}$ and $t$ as parameters. The averaging operator of $f$ is defined by

$\langle f\rangle(x)=\lambda^{-1} \int_{x-\lambda / 2}^{x+\lambda / 2} f(\xi) d \xi$,

$x \in[\lambda / 2, H-\lambda / 2]$,

where $\lambda$ is the constant thickness of every lamina. It can be shown (cf. the book edited by Woźniak Cz., Michalak and Jędrysiak [29]) that for toleranceperiodic function $f$ of $x$, its averaged value calculated from (2) is a slowly-varying function in $x$.

A continuous function $F$, defined on $\bar{\Omega}$, is called slowly-varying (with tolerance parameter $d_{0}>0$ ) if $\forall x \in \bar{\Omega}[F]_{x} \leq d_{0}$, and we will write $F \in S V^{0}(\square) \subset C^{0}(\bar{\Omega})$. Denote $\nabla^{(r)} F \equiv \partial^{r} F / \partial x^{r}$, $\nabla^{(0)} F \equiv F$. Function $F \in C^{1}(\bar{\Omega})$ is called slowlyvarying of the first kind with tolerance given by $d=\left(d_{0}, d_{1}\right)$ (shortly slowly-varying) and denoted as $F \in S V^{1}(\square) \subset C^{1}(\bar{\Omega})$ if

(i) $\nabla^{(r)} F \in S V^{0}(\square), r=0,1$;

(ii) $(\forall x \in \bar{\Omega})\left[\lambda\left|\partial\left(\nabla^{(0)} F(x)\right)\right| \leq d_{1}\right]$.

Let $f$ be an integrable, bounded function in $\bar{\Omega}$. The function $f$ is called the tolerance-periodic function, $T P(\square)$, if for every $x \in \bar{\Omega}$ there exists $\square$-periodic function $f_{x}$ such that $f \mid \square(x) \cap \operatorname{Dom} f$ and $f_{x} \mid \square(x) \cap \operatorname{Dom} f_{x}$ are indiscernible in tolerance determined by $(d, \lambda)$. In this case every $f_{x}$ is called the periodic approximation of $f$ in $\square(x)$.

Denote a tolerance-periodic continuous function defined on $\bar{\Omega}$ by $\varphi(\cdot)$. Its gradient $\nabla^{(1)} \varphi$ is a piecewise continuous and bounded. Function $\varphi(\cdot)$ is called the fluctuation shape function (FSF) of the first kind, if it depends on $\lambda$ as a parameter and satisfies conditions:

(i) $\partial^{r} \varphi \in O\left(\lambda^{R-r}\right)$ for $r=0,1, \ldots, R, R=1$, $\partial^{0} \varphi \equiv \varphi$

(ii) $\langle\varphi\rangle(x) \approx 0$ for every $x \in \Omega$;

(iii) $\nabla^{1} \varphi \approx \partial^{1} \varphi$, for $\forall x \in \Omega$.

Denote set of all fluctuation shape functions of the first kind by $F S^{1}(\square)$. Condition (ii) can be replaced by $\langle c \varphi\rangle(x) \approx 0$ for every $x \in \Omega$, where $c$ is a certain positive tolerance-periodic function.
The above concepts were introduced in the book by Woźniak Cz. and Wierzbicki [30], however the detailed discussion of them can be found in the monography edited by Woźniak Cz., Michalak and Jędrysiak [29].

\subsection{Modelling assumptions}

The modelling procedure of the tolerance averaging technique is based on the additional assumptions, which were formulated in the general form in the book edited by Woźniak Cz., Michalak and Jędrysiak [29].

The first modelling assumption is the micro-macro decomposition, which states that the temperature field $T=T(x, \mathbf{x}, t), x \in \bar{\Omega}, \mathbf{x} \in \bar{\Xi}$, is restricted by the following formula

$T(x, \mathbf{x}, t)=\vartheta(x, \mathbf{x}, t)+\varphi(x) \psi(x, \mathbf{x}, t)$,

where $\vartheta(\cdot, \mathbf{x}, t) \in S V^{1}(\square)$ is called the macrotemperature, $\psi(\cdot, \mathbf{x}, t) \in S V^{1}(\square)$ is called the amplitude fluctuation of the temperature. Functions $\vartheta(\cdot), \psi(\cdot)$ are the basic unknowns, which describe a distribution of the temperature in the TGL layer.

Following Jędrysiak and Radzikowska [10] and the book edited by Woźniak Cz., Michalak and Jędrysiak [29] we assume the fluctuation shape function $\varphi(x)$, $x \in \bar{\Omega}$, to be continuous, linear across every sublamina thickness and of an order $O(\lambda)$, and to satisfy the pertinent conditions (i)-(iii). Hence, this function can be written in the following form:

$\varphi(x)=\left\{\begin{array}{l}-\lambda \sqrt{3} \frac{v(\bar{x})}{v^{\prime \prime}(\bar{x})}\left[2 \frac{x}{\lambda}+v^{\prime}(\bar{x})\right] \\ \quad \text { for } x \in\left(-\lambda / 2,-\lambda / 2+\lambda v^{\prime \prime}(\bar{x})\right), \\ \lambda \sqrt{3} \frac{v(\bar{x})}{v^{\prime}(\bar{x})}\left[2 \frac{x}{\lambda}-v^{\prime \prime}(\bar{x})\right] \\ \quad \text { for } x \in\left(\lambda / 2-\lambda v^{\prime}(\bar{x}), \lambda / 2\right),\end{array}\right.$

where $\bar{x}$ is a centre of interval $\square=(-\lambda / 2, \lambda / 2)$; cf. Fig. 2. Moreover, function $\varphi$ has values $\lambda \sqrt{3} v(\bar{x})$ at the interfaces between laminas and values $-\lambda \sqrt{3} v(\bar{x})$ at the interfaces between adjacent sub-laminas within the lamina. Because the non-homogeneity ratio $v(\cdot)$ is a slowly-varying function it can be shown that the mean value of $\varphi$ in every lamina is equal zero.

The second assumption, called the tolerance averaging approximation (TAA), states that in the mod- 


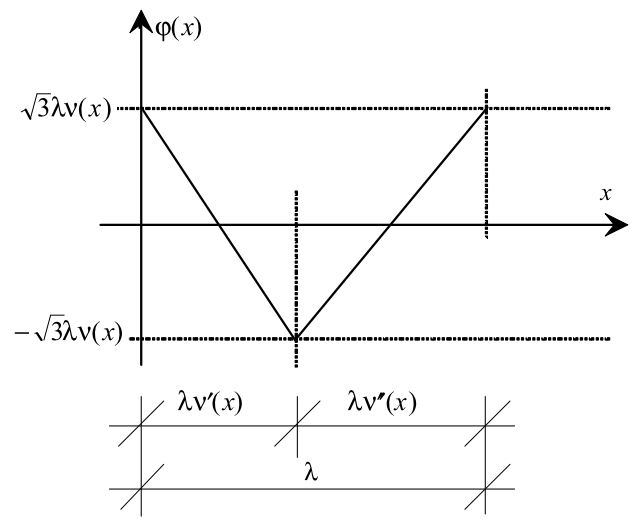

Fig. 2 Scheme of the fluctuation shape function

elling terms $O(d)$ are negligibly small, e.g. in formulas:

$$
\begin{aligned}
& \langle f\rangle(x)=\langle\bar{f}\rangle(x)+O(d), \\
& \langle f F\rangle(x)=\langle f\rangle(x) F(x)+O(d), \\
& \langle f \partial(\varphi F)\rangle(x)=\langle f \partial \varphi\rangle(x) F(x)+O(d), \\
& x \in \Omega ; \quad 0<d \ll 1 ; \quad f \in T P(\square), \\
& F \in S V^{1}(\square), \quad \varphi \in F S^{1}(\square) .
\end{aligned}
$$

\subsection{Modelling procedure}

The modelling procedure for TGL-type composites is similar to the procedure of the tolerance averaging technique shown in Woźniak Cz. and Wierzbicki [30]. This procedure is generalised in the book edited by Woźniak Cz., Michalak and Jędrysiak [29]. Following this book the modelling procedure will be outlined.

Firstly, the starting point of the modelling is the Fourier's heat conduction equation (1). Following the aforementioned book we can introduce the polynomial

$\mathcal{L}=\frac{1}{2}(\tau c \dot{T} \dot{T}+\nabla T \cdot \mathbf{K} \cdot \nabla T)+f T$,

with $\tau$ as a time-dimensional constant. It can be shown that (5) generates the equation

$\nabla \cdot \frac{\partial \mathcal{L}}{\partial(\nabla T)}-\frac{1}{\tau} \frac{\partial \mathcal{L}}{\partial \dot{T}}-\frac{\partial \mathcal{L}}{\partial T}=0$.

Equation (6) coincides with the heat conduction equation (1).

Secondly, the tolerance averaging is applied to (1). Substituting the right-hand side of (3) into (5) and averaging polynomial $\mathcal{L}$, using (2) and $T A A$, we arrive at the averaged polynomial in the form

$$
\begin{aligned}
\langle\mathcal{L}\rangle= & \frac{1}{2}(\nabla \vartheta \cdot\langle\mathbf{K}\rangle \cdot \nabla \vartheta+\psi\langle\partial \varphi \cdot \mathbf{K} \cdot \partial \varphi\rangle \psi \\
& +\bar{\nabla} \psi \cdot\left\langle\mathbf{K} \varphi^{2}\right\rangle \cdot \bar{\nabla} \psi+\nabla \vartheta \cdot\langle\mathbf{K} \cdot \partial \varphi\rangle \psi \\
& +\psi\langle\partial \varphi \cdot \mathbf{K}\rangle \cdot \nabla \vartheta+\tau\langle c\rangle \dot{\vartheta} \dot{\vartheta} \\
& \left.+\tau\left\langle c \varphi^{2}\right\rangle \dot{\psi} \dot{\psi}\right)+\langle f\rangle \vartheta+\langle f \varphi\rangle \psi .
\end{aligned}
$$

The tolerance averaging of (6) yields the following system of equations:

$\nabla \cdot \frac{\partial\langle\mathcal{L}\rangle}{\partial \nabla \vartheta}-\frac{1}{\tau} \frac{\partial\langle\mathcal{L}\rangle}{\partial \dot{\vartheta}}-\frac{\partial\langle\mathcal{L}\rangle}{\partial \vartheta}=0$,

$\bar{\nabla} \cdot \frac{\partial\langle\mathcal{L}\rangle}{\partial \bar{\nabla} \psi}-\frac{1}{\tau} \frac{\partial\langle\mathcal{L}\rangle}{\partial \dot{\psi}}-\frac{\partial\langle\mathcal{L}\rangle}{\partial \psi}=0$,

which have slowly-varying coefficients.

\section{Governing equations of averaged model}

Denote $\mathbf{e}=(1,0,0)$ and introduce the smooth functional coefficients:

$\langle c\rangle=v^{\prime} c^{\prime}+v^{\prime \prime} c^{\prime \prime}, \quad\langle\mathbf{K}\rangle=v^{\prime} \mathbf{K}^{\prime}+v^{\prime \prime} \mathbf{K}^{\prime \prime}$,

$[\mathbf{K}]=2 \sqrt{3} v \mathbf{e} \cdot\left(\mathbf{K}^{\prime}-\mathbf{K}^{\prime \prime}\right)$,

$[\mathbf{K}]^{T}=2 \sqrt{3} v\left(\mathbf{K}^{\prime}-\mathbf{K}^{\prime \prime}\right) \cdot \mathbf{e}$,

$\{K\}=12 \mathbf{e} \cdot\left(v^{\prime} \mathbf{K}^{\prime \prime}+v^{\prime \prime} \mathbf{K}^{\prime}\right) \cdot \mathbf{e}$,

where $v^{\prime}=v^{\prime}(x), v^{\prime \prime}=1-v^{\prime \prime}(x)$, and $c^{\prime}, c^{\prime \prime}$ as well as $\mathbf{K}^{\prime}, \mathbf{K}^{\prime \prime}$ are constant. Combining (8) with (7), bearing in mind that $\langle\mathbf{K} \varphi\rangle=0,\langle c \varphi\rangle=0$ and using the above denotations we arrive to the following form of averaged heat conduction equations:

$$
\begin{aligned}
& \nabla \cdot(\langle\mathbf{K}\rangle \cdot \nabla \vartheta+[\mathbf{K}] \psi)-\langle c\rangle \dot{\vartheta}=\langle f\rangle, \\
& \lambda^{2} \nu^{2}[\bar{\nabla} \cdot(\langle\mathbf{K}\rangle \cdot \bar{\nabla} \psi)-\langle c\rangle \dot{\psi}] \\
& \quad-\{K\} \psi-[\mathbf{K}]^{T} \cdot \nabla \vartheta=\langle f \varphi\rangle .
\end{aligned}
$$

It can be observed that (10) have coefficients being smooth, slowly-varying functions in $x$ in contrast to (1) with discontinuous, highly oscillating coefficients. Some terms of the above equations depend on the microstructure parameter $\lambda$. Hence, the proposed model describes the effect of the microstructure size of the TGL-type composite (e.g. a TGL layer) on the heat conduction. Moreover, we have to formulate two boundary conditions for the macrotemperature $\vartheta$ with 
respect to $x_{i}(i=1,2,3)$ and also two boundary conditions for the amplitude fluctuation $\psi$ with respect to $x$.

Summing up, (10) together with micro-macro decomposition (3) and certain applicability conditions for the basic unknowns, i.e. $\vartheta(\cdot, \mathbf{x}, t)$, $\psi(\cdot, \mathbf{x}, t) \in S V^{1}(\square)$, constitute the tolerance model of the heat conduction in transversally graded laminate.

The conditions for the basic unknowns can be used for a posteriori evaluation of tolerance parameter $d, d \ll 1$, and hence for the verification of the physical reliability of obtained solutions. Moreover, the temperature field of the TGL-type composite can be approximated by means of formula (3) with the fluctuation shape function $\varphi$ in the form (4).

\section{Applications to a stationary heat conduction across a TGL layer}

Let us consider a TGL layer subjected to a thermal gradient in the direction parallel to the $x$-axis. Moreover, we assume that the problem of the heat conduction is stationary. Hence, the temperature field $T$ is a function of argument $x$, i.e. $T=T(x)$. Let us denote by $k \equiv k_{11}, k^{\prime} \equiv k_{11}^{\prime}, k^{\prime \prime} \equiv k_{11}^{\prime \prime}$ heat conduction coefficients in sub-laminas.

\subsection{Heat conduction within the tolerance model}

Since we analyse the stationary heat conduction along the $x$-axis, the basic unknowns in the tolerance model are functions of argument $x$, i.e. $\vartheta=\vartheta(x), \psi=\psi(x)$. Introducing notations:

$$
\begin{aligned}
& K \equiv v^{\prime} k^{\prime}+v^{\prime \prime} k^{\prime \prime}, \quad \tilde{K} \equiv 2 \sqrt{3} v\left(k^{\prime}-k^{\prime \prime}\right), \\
& \breve{K} \equiv 12\left(v^{\prime} k^{\prime \prime}+v^{\prime \prime} k^{\prime}\right),
\end{aligned}
$$

and assuming that heat sources are omitted, i.e. $f=0$, equations (10) for the problem under consideration take the form:

$\partial[K(x) \partial \vartheta+\tilde{K}(x) \psi]=0$,

$\tilde{K}(x) \partial \vartheta+\breve{K}(x) \psi=0$.

It can be observed that the amplitude fluctuation $\psi$ can be calculated from $(12)_{2}$ :

$\psi=-\tilde{K}(x)[\breve{K}(x)]^{-1} \partial \vartheta$.
Substituting the right-hand side of formula (13) into (12) 1 and denoting

$K^{e f f}(x) \equiv K(x)-[\tilde{K}(x)]^{2}[\breve{K}(x)]^{-1}$

we can write the following equations:

$\partial\left[K^{e f f}(x) \partial \vartheta\right]=0$,

$\psi=-\tilde{K}(x)[\breve{K}(x)]^{-1} \partial \vartheta$.

Equations (15) together with micro-macro decomposition (3), which takes the form

$T(x)=\vartheta(x)+\varphi(x) \psi(x)$,

describe the stationary heat conduction across laminas in the TGL layer.

The above equations have slowly-varying functional coefficients. However, these coefficients are defined by known functions, e.g. by the fraction ratios of materials $v^{\prime}, v^{\prime \prime}$, the non-homogeneity ratio $v$, the fluctuation shape function $\varphi$, cf. (4). Hence, (15) 1 can be solved analytically by integrating this equation. Solution to $(15)_{1}$ can be written as:

$\vartheta(x)=C_{1} \int\left[K^{e f f}(x)\right]^{-1} d x+C_{2}$,

where $C_{1}, C_{2}$ are constants determined by boundary conditions. For the layer under consideration with constant heat conduction coefficients $k^{\prime}, k^{\prime \prime}$ and the fluctuation shape function given by (4) the averaged heat conduction coefficient $K^{e f f}$, (14), is equal

$K^{e f f}(x)=k^{\prime} k^{\prime \prime}\left[k^{\prime}+\left(k^{\prime \prime}-k^{\prime}\right) v^{\prime}(x)\right]^{-1}$.

Denoting

$N(x)=\int v^{\prime}(x) d x$

the macrotemperature (17) takes the form

$\vartheta(x)=C_{1}\left[x\left(k^{\prime \prime}\right)^{-1}+\left(k^{\prime \prime}-k^{\prime}\right)\left(k^{\prime} k^{\prime \prime}\right)^{-1} N(x)\right]+C_{2}$.

For the macrotemperature $\vartheta$ we formulate the boundary conditions in the form

$x=0: \quad \vartheta(0)=T_{0} ; \quad x=H: \quad \vartheta(H)=0$. 


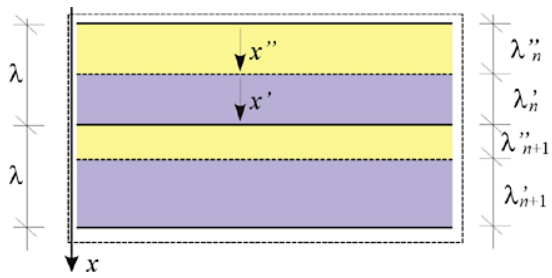

Fig. 3 A fragment of the TGL layer with local coordinates $x^{\prime}, x^{\prime \prime}$

Combining the boundary conditions (21) with formula (20) constants $C_{1}, C_{2}$ can be calculated from the system of algebraic linear equations:

$C_{1}=T_{0} \frac{k^{\prime} k^{\prime \prime}}{\left(k^{\prime \prime}-k^{\prime}\right)[N(0)-N(H)]-k^{\prime} H}$,
$C_{2}=-T_{0} \frac{k^{\prime} H+\left(k^{\prime \prime}-k^{\prime}\right) N(H)}{\left(k^{\prime \prime}-k^{\prime}\right)[N(0)-N(H)]-k^{\prime} H}$.

Substituting the right-hand side of formula (20) into $(15)_{2}$ we obtain the formula for the amplitude fluctuation $\psi$ :

$\psi=\frac{\sqrt{3}}{6} \frac{k^{\prime \prime}-k^{\prime}}{k^{\prime} k^{\prime \prime}} v(x) C_{1}$.

Combining (23) and solution (20) with formula (16) the temperature takes the form:

$$
\begin{aligned}
T(x)= & \left\{\frac{x}{k^{\prime \prime}}+\frac{k^{\prime \prime}-k^{\prime}}{k^{\prime} k^{\prime \prime}}[N(x)\right. \\
& \left.\left.+\frac{\sqrt{3}}{6} \varphi(x) v(x)\right]\right\} C_{1}+C_{2},
\end{aligned}
$$

with constants $C_{1}, C_{2}$ determined by (22). Formula (24) describes the "exact" distribution of the temperature in the TGL layer by the tolerance model.

\subsection{Heat conduction by the higher-order theory (Aboudi, Pindera and Arnold [2])}

In this subsection the stationary heat conduction is analysed in the framework of the higher-order theory. This modelling technique was used in a series of papers and discussed in the paper by Aboudi, Pindera and Arnold [1]. Following this paper the outline of the modelling procedure of this theory is shown below.

Let $x^{\prime}, x^{\prime \prime}$ be local coordinates in sub-laminas in the $n^{\text {th }}$ lamina $(n=1, \ldots, m)$, cf. Fig. 3 . The main assumption of this modelling approach is that the temperature distributions in both the materials in the $n^{\text {th }}$ lamina are postulated in the following form:

$$
\begin{aligned}
& T^{\prime}\left(x^{\prime}\right)=T_{0}^{\prime}+x^{\prime} T_{1}^{\prime}+\frac{1}{2}\left[3\left(x^{\prime}\right)^{2}-\left(\frac{1}{2} \lambda_{n}^{\prime}\right)^{2}\right] T_{2}^{\prime}, \\
& T^{\prime \prime}\left(x^{\prime \prime}\right)=T_{0}^{\prime \prime}+x^{\prime \prime} T_{1}^{\prime \prime}+\frac{1}{2}\left[3\left(x^{\prime \prime}\right)^{2}-\left(\frac{1}{2} \lambda_{n}^{\prime \prime}\right)^{2}\right] T_{2}^{\prime \prime},
\end{aligned}
$$

where parameters $T_{0}^{\prime}, T_{1}^{\prime}, T_{2}^{\prime}, T_{0}^{\prime \prime}, T_{1}^{\prime \prime}, T_{2}^{\prime \prime}$ are unknown constants. It can be observed that in the case of the layer made of $m$ laminas we have $6 m$ basic unknowns of the model. Hence, in order to calculate these unknowns we have to formulate $6 \mathrm{~m}$ additional conditions.

Using the aforementioned denotations for heat conduction coefficients in sub-laminas: $k^{\prime}, k^{\prime \prime}$; and for the derivative of $x=x^{\prime}, x^{\prime \prime}: \partial \equiv \partial / \partial x$, we can write these additional relations below:

- the heat conduction equations for the $n^{\text {th }}$ lamina

$$
\begin{aligned}
& -\partial\left[k^{\prime} \partial T^{\prime}\left(x^{\prime}\right)\right]=0, \\
& -\partial\left[k^{\prime \prime} \partial T^{\prime \prime}\left(x^{\prime \prime}\right)\right]=0,
\end{aligned}
$$

- the continuity conditions of heat fluxes for the $n^{\text {th }}$ lamina

$$
\begin{aligned}
& \left.k^{\prime} \partial T^{\prime}\right|_{x^{\prime}=\frac{1}{2} \lambda_{n}^{\prime}} ^{n}=\left.k^{\prime \prime} \partial T^{\prime \prime}\right|_{x^{\prime \prime}=-\frac{1}{2} \lambda_{n}^{\prime \prime}} ^{n}, \\
& \left.k^{\prime} \partial T^{\prime}\right|_{x^{\prime}=-\frac{1}{2} \lambda_{n+1}^{\prime}} ^{n+1}=\left.k^{\prime \prime} \partial T^{\prime \prime}\right|_{x^{\prime \prime}=\frac{1}{2} \lambda_{n}^{\prime \prime}} ^{n},
\end{aligned}
$$

- the continuity conditions of the temperature for the $n^{\text {th }}$ lamina

$$
\begin{aligned}
& \left.T^{\prime}\right|_{x^{\prime}=\frac{1}{2} \lambda_{n}^{\prime}} ^{n}=\left.T^{\prime \prime}\right|_{x^{\prime \prime}=-\frac{1}{2} \lambda_{n}^{\prime \prime}} ^{n}, \\
& \left.T^{\prime}\right|_{x^{\prime}=-\frac{1}{2} \lambda_{n+1}^{\prime}} ^{n+1}=\left.T^{\prime \prime}\right|_{x^{\prime \prime}=\frac{1}{2} \lambda_{n}^{\prime \prime}} ^{n},
\end{aligned}
$$

- the boundary conditions (assumed here in the form of (21))

$$
\begin{aligned}
& \left.T^{\prime}\right|^{1}=T_{1}=T_{0}, \\
& x^{\prime}=-\frac{1}{2} \lambda_{1}^{\prime} \quad(\text { i.e. } x=0), \\
& \left.T^{\prime \prime}\right|^{m}=T_{m}=0, \\
& \left.x^{\prime \prime}=\frac{1}{2} \lambda_{m}^{\prime \prime} \quad \text { (i.e. } x=H\right),
\end{aligned}
$$

where $T_{1}$ and $T_{m}$ are temperatures on boundaries $x=0$ and $x=H$, respectively. 


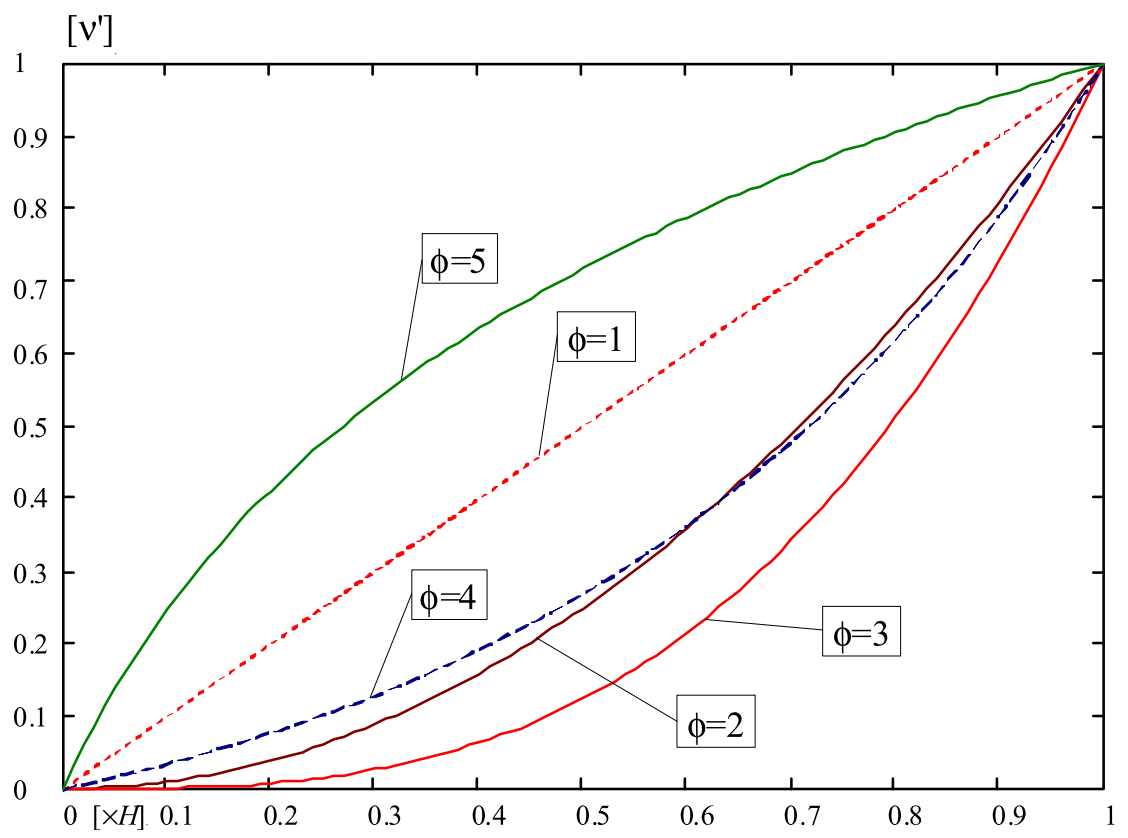

Fig. 4 The fraction ratios of materials $v^{\prime}$ versus coordinate $x(\phi=1$-for formulas (30), $\phi=2$-for formulas (31), $\phi=3$-for formulas (32), $\phi=4$-for formulas (33), $\phi=5$-for formulas (34))

Combining relations (26)-(29) with distributions (25) we arrive at a system of $6 \mathrm{~m}$ algebraic linear equations for $6 m$ basic unknowns $T_{0}^{\prime}, T_{1}^{\prime}, T_{2}^{\prime}, T_{0}^{\prime \prime}, T_{1}^{\prime \prime}, T_{2}^{\prime \prime}$.

Some calculational results illustrating both models are shown in the subsequent section.

\section{Results}

Let us consider five different cases of the material distribution across the layer thickness assumed in the form of:

1) the linear functions (denoted by number $\phi=1$ )

$$
v^{\prime}(x)=x / H, \quad v^{\prime \prime}(x)=1-v^{\prime}(x) ;
$$

2) the square functions (denoted by $\phi=2$ )

$$
v^{\prime}(x)=(x / H)^{2}, \quad v^{\prime \prime}(x)=1-v^{\prime}(x) ;
$$

3) the cubic functions (denoted by $\phi=3$ )

$$
v^{\prime}(x)=(x / H)^{3}, \quad v^{\prime \prime}(x)=1-v^{\prime}(x) ;
$$

4) the exponential functions (denoted by $\phi=4$ )

$$
\begin{aligned}
& v^{\prime}(x)=[1-\exp (2 x / H)][1-\exp (2)]^{-1}, \\
& v^{\prime \prime}(x)=1-v^{\prime}(x) ;
\end{aligned}
$$

5) the logarithmic functions (denoted by $\phi=5$ )

$$
\begin{aligned}
& v^{\prime}(x)=\frac{1}{2} \ln [(\exp (2)-1)(x / H)+1], \\
& v^{\prime \prime}(x)=1-v^{\prime}(x) .
\end{aligned}
$$

Functions $v^{\prime}(\cdot)$ are presented in Fig. 4.

Some calculational results are shown in Figs. 5-10. In Figs. 5a-b there are presented plots of distributions of the temperature given by formula (24), i.e. the temperature by the tolerance model versus coordinate $x \in[0, H]$. These curves are made for ratio $\lambda / H=0.02$ and the fraction ratios of materials (30)-(34) (denoted by $\phi=1, \ldots, 5)$. Figure 5a shows diagrams for ratios $k^{\prime \prime} / k^{\prime}=1 / 2$ (a) and $k^{\prime \prime} / k^{\prime}=2$ (b), but Fig. 5b-for ratios $k^{\prime \prime} / k^{\prime}=1 / 16$ (a) and $k^{\prime \prime} / k^{\prime}=16$ (b). Figures 6-10 present comparisons between distributions of the temperature obtained in the framework of the tolerance model (TM) and the higher-order theory (HT). In Figs. 6, 7, 8, 9, 10 there are plots made for the fraction ratios of materials: linear (30), square (31), cubic (32), exponential (33), logarithmic (34), respectively. These diagrams are calculated for ratios: $\lambda / H=0.02, k^{\prime \prime} / k^{\prime}=1 / 16$ (a), $k^{\prime \prime} / k^{\prime}=16$ (b), $k^{\prime \prime} / k^{\prime}=1 / 2$ (c) and $k^{\prime \prime} / k^{\prime}=2$ (d). Results are shown in two intervals of argument $x$ : 

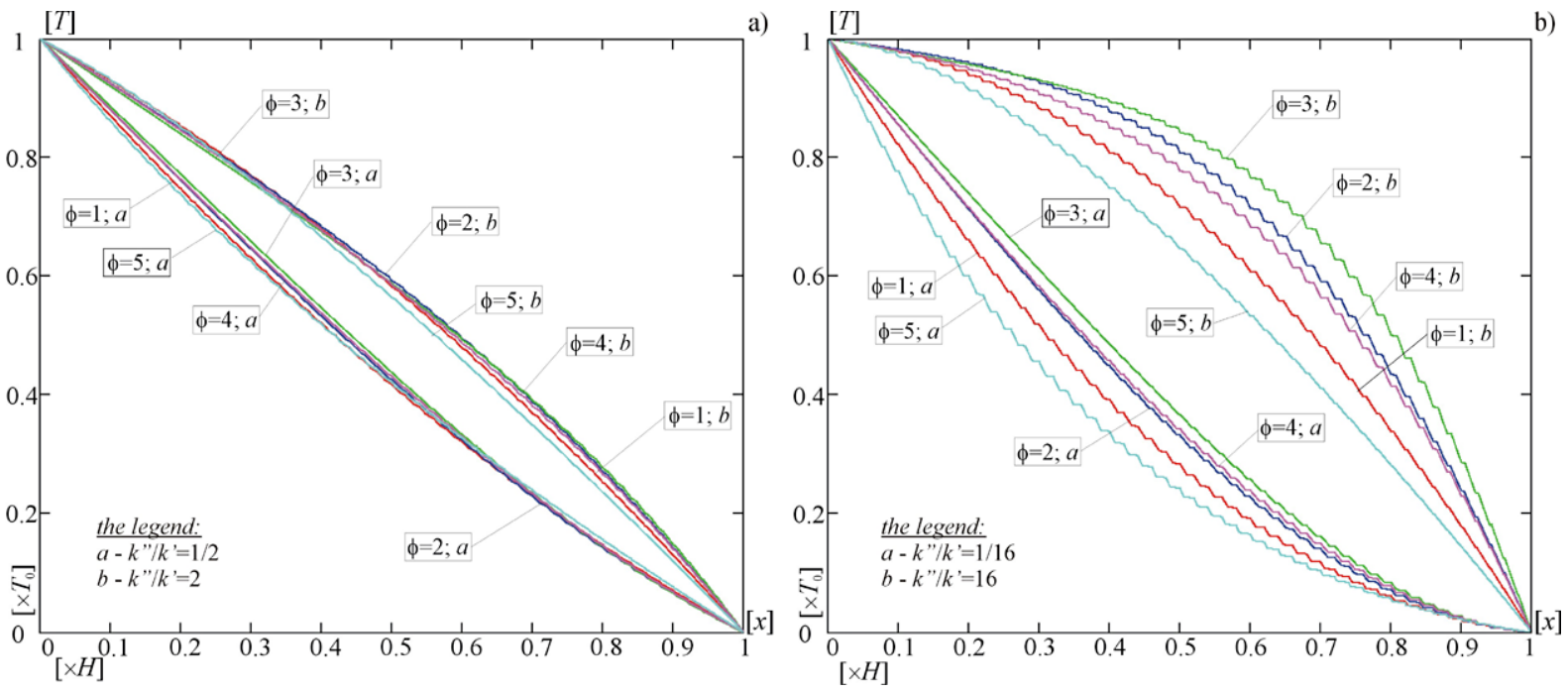

Fig. 5 Diagrams of temperature $T$ across the TGL layer by the tolerance model: (a) for ratios $k^{\prime \prime} / k^{\prime}=1 / 2$ (a) and $k^{\prime \prime} / k^{\prime}=2$ (b); (b) for ratios $k^{\prime \prime} / k^{\prime}=1 / 16$ (a) and $k^{\prime \prime} / k^{\prime}=16$ (b) (for ratio $\lambda / H=0.02$; for the fraction ratios of materials (30)-(34) $(\phi=1, \ldots, 5)$ )
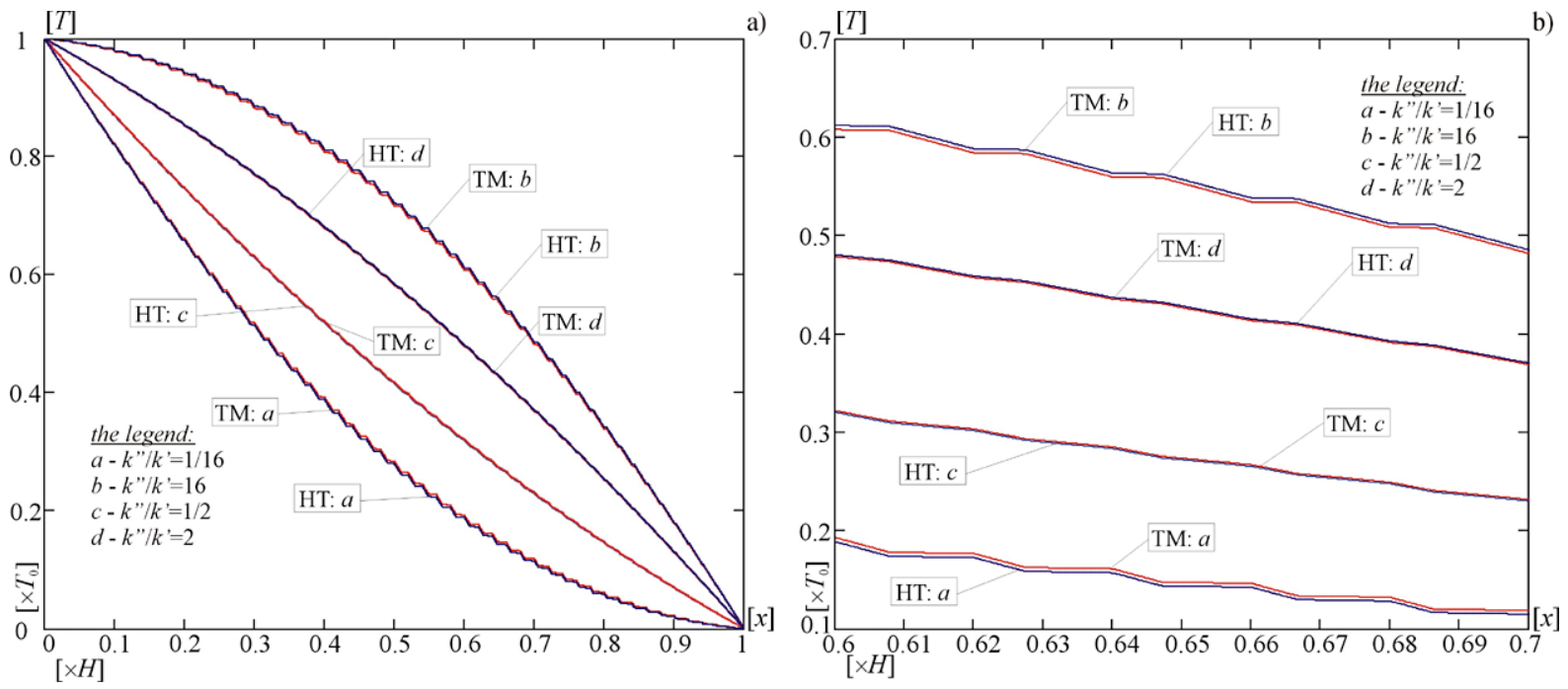

Fig. 6 Diagrams of temperature $T$ by the tolerance model (TM) and the higher-order theory (HT) for the fraction ratios of materials (30) $(\phi=1)$ : (a) for interval $[0, H]$; (b) for interval $[0.6 H, 0.7 H]$ (for ratio $\lambda / H=0.02)$

[0,H]-Figs. 6a, 7a, 8a, 9a, 10a; $[0.6 H, 0.7 H]-$ Figs. 6b, 7b, 8b, 9b, $10 \mathrm{~b}$.

Analysing obtained results some remarks can be formulated.

- Functions of the temperature in the TGL layer, which are obtained in the framework of the tolerance model, take into account the effect of the microstructure, cf. Figs. 5-10.
- Distributions of the temperature depend on distributions of material properties of the TGL layer, cf. Fig. 5, in such a way that values of the temperature decrease for the following sequence of the fraction ratios of materials: logarithmic, linear, exponential, square and cubic for arguments $x$ close to 0 if $k^{\prime \prime}>k^{\prime}$ and $x$ close to $H$ if $k^{\prime \prime}<k^{\prime}$. However, values of the temperature decrease for the following sequence of the fraction ratios of materials: cubic, 

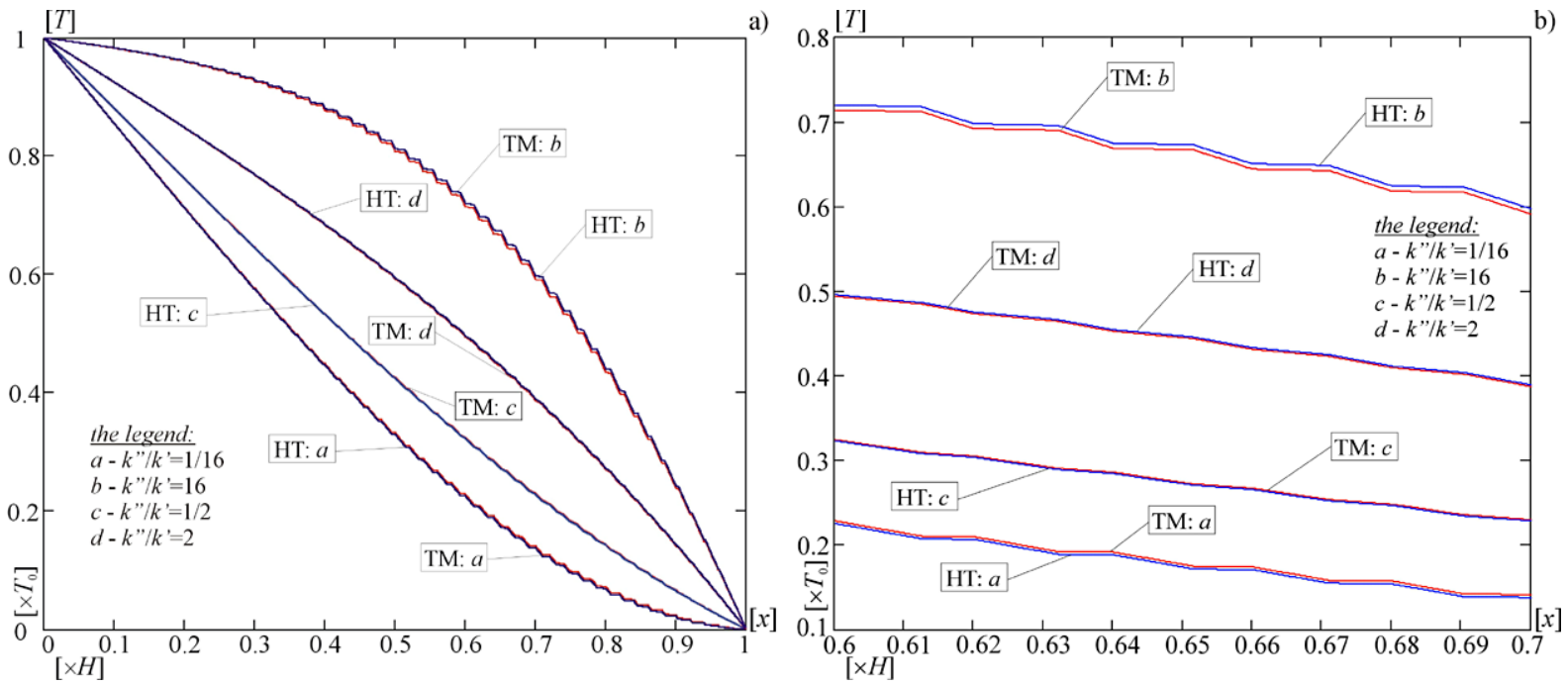

Fig. 7 Diagrams of temperature $T$ by the tolerance model (TM) and the higher-order theory (HT) for the fraction ratios of materials (31) $(\phi=2)$ : (a) for interval $[0, H]$; (b) for interval $[0.6 H, 0.7 H]$ (for ratio $\lambda / H=0.02$ )
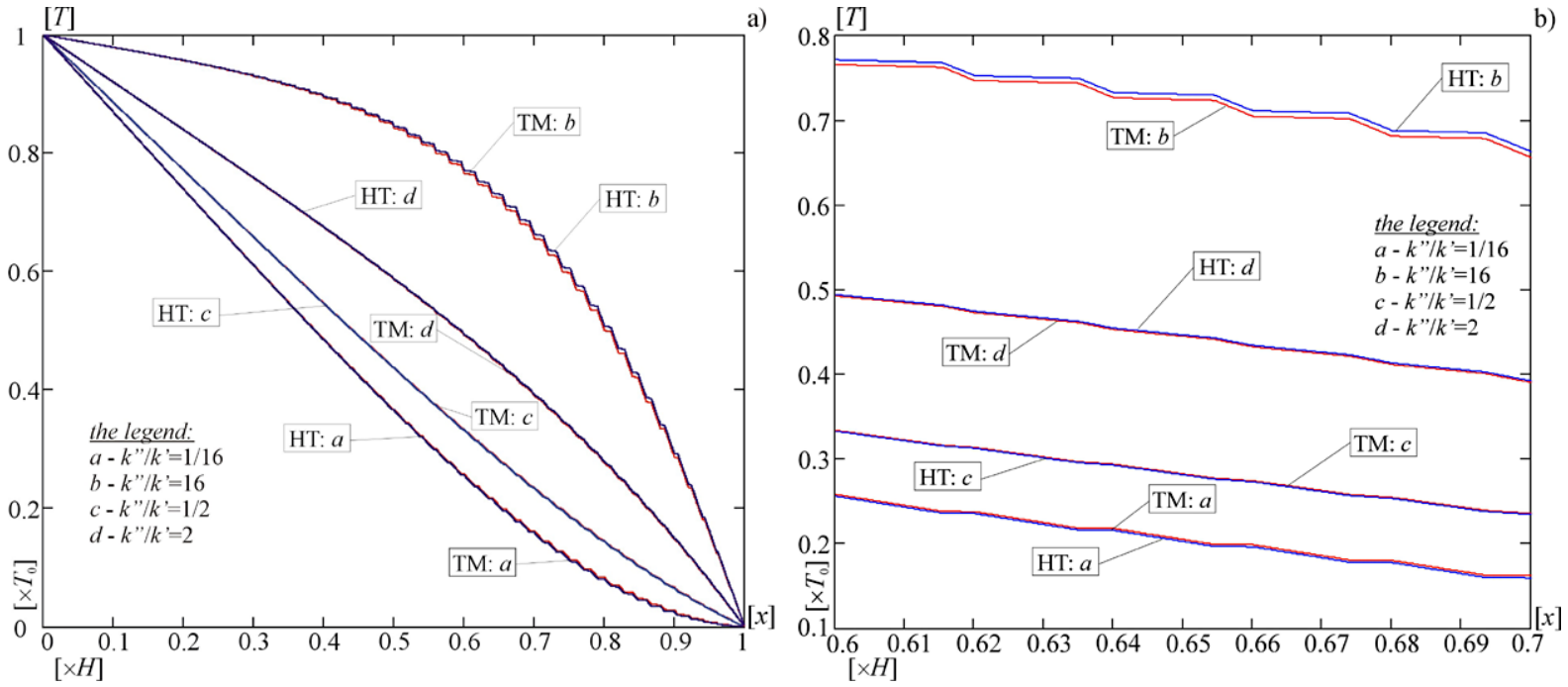

Fig. 8 Diagrams of temperature $T$ by the tolerance model (TM) and the higher-order theory (HT) for the fraction ratios of materials (32) $(\phi=3)$ : (a) for interval $[0, H]$; (b) for interval $[0.6 H, 0.7 H]$ (for ratio $\lambda / H=0.02$ )

square, exponential, linear and logarithmic for arguments $x$ close to 0 if $k^{\prime \prime}<k^{\prime}$ and $x$ close to $H$ if $k^{\prime \prime}>k^{\prime}$.

- Distributions of the temperature strikingly depend on differences between values of heat conduction coefficients $k^{\prime}, k^{\prime \prime}$ of material properties of the TGL layer. Values of the temperature for the same fraction ratios of materials are smaller for $k^{\prime \prime}<k^{\prime}$ (e.g. $k^{\prime \prime} / k^{\prime}=1 / 2 ; 1 / 16$ ) than for $k^{\prime \prime}>k^{\prime}$ (e.g. $k^{\prime \prime} / k^{\prime}=2$; 16), cf. Fig. 5. In addition, the temperature decreases more strongly for cases $k^{\prime \prime}<k^{\prime}$ than for cases $k^{\prime \prime}>k^{\prime}$ and this decrease in temperature is stronger for $k^{\prime \prime} / k^{\prime} \rightarrow 0$, cf. Fig. 5b. However, for small differences between heat conduction coefficients, e.g. $k^{\prime \prime} / k^{\prime}=1 / 2 ; 2$, cf. Fig. 5a, differences between distributions of the temperature for various fraction ratios of materials are smaller than for big differences between heat con- 

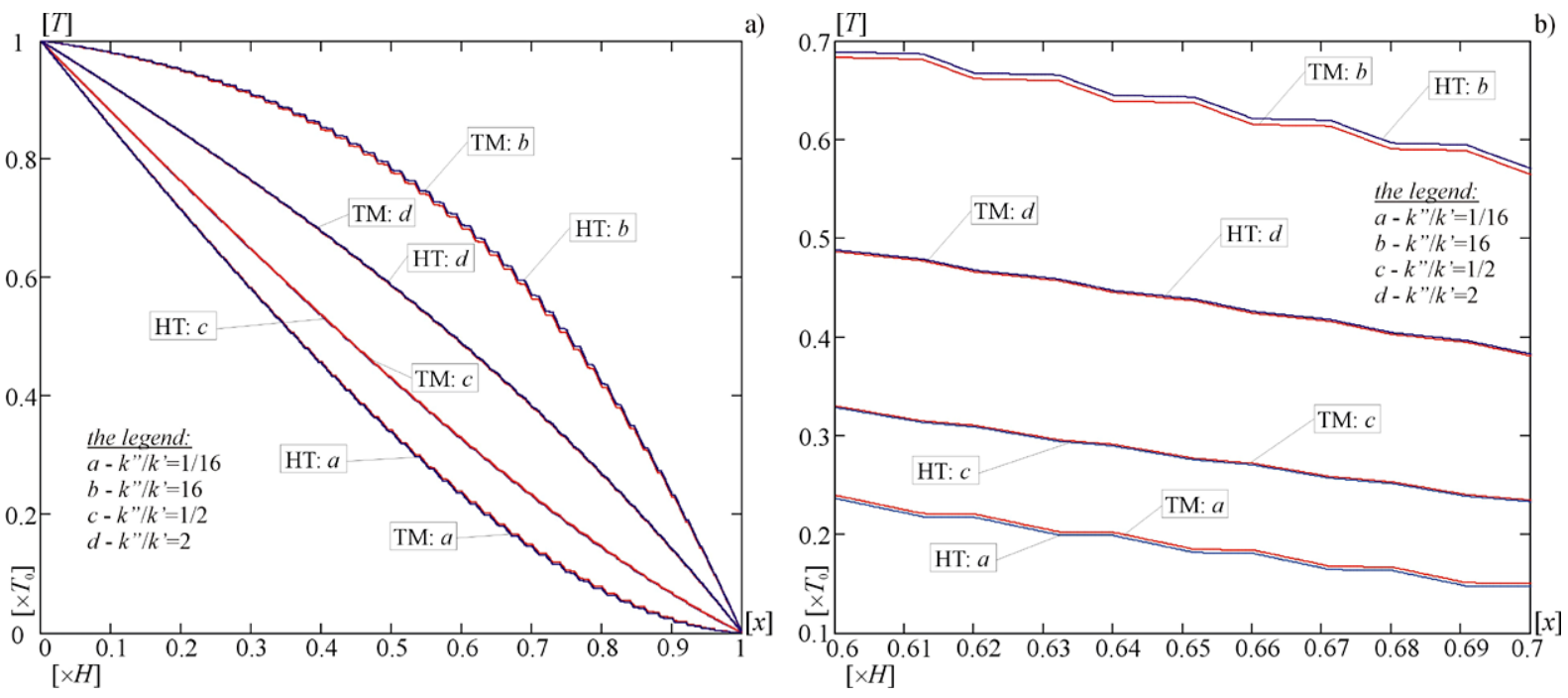

Fig. 9 Diagrams of temperature $T$ by the tolerance model (TM) and the higher-order theory (HT) for the fraction ratios of materials (33) $(\phi=4)$ : (a) for interval $[0, H]$; (b) for interval $[0.6 H, 0.7 H]$ (for ratio $\lambda / H=0.02$ )
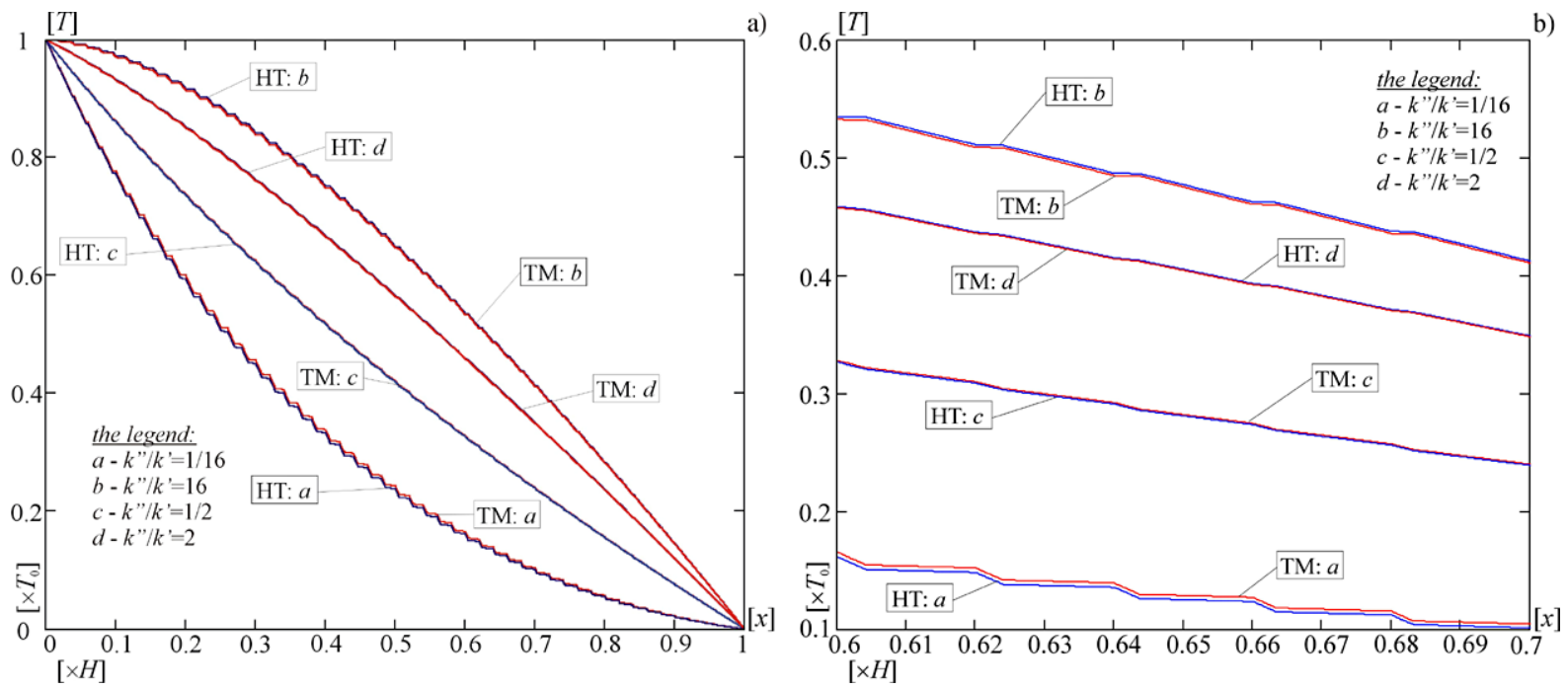

Fig. 10 Diagrams of temperature $T$ by the tolerance model (TM) and the higher-order theory (HT) for the fraction ratios of materials (34) $(\phi=5)$ : (a) for interval $[0, H]$; (b) for interval $[0.6 H, 0.7 H]$ (for ratio $\lambda / H=0.02$ )

duction coefficients, e.g. $k^{\prime \prime} / k^{\prime}=1 / 16 ; 16$, cf. Fig. 5b.

- Differences between distributions of the temperature calculated by using the tolerance model (TM) and the higher-order theory (HT) are very small; it is shown in Figs. 6-10.

- Values of the temperature obtained in the framework of the tolerance model are higher than values of the temperature from the higher-order the- ory for $k^{\prime \prime}<k^{\prime}$ (e.g. $\left.k^{\prime \prime} / k^{\prime}=1 / 2 ; 1 / 16\right)$ whereas they are smaller than values of the temperature from the higher-order theory for $k^{\prime \prime}>k^{\prime}$ (e.g. $\left.k^{\prime \prime} / k^{\prime}=2 ; 16\right)$.

- It can be observed that for the analysed fraction ratios of materials differences between values of the temperature obtained within the tolerance model and the higher-order theory can be put in the following decreasing order for $k^{\prime \prime}<k^{\prime}$ 
(e.g. $k^{\prime \prime} / k^{\prime}=1 / 2 ; 1 / 16$ ): for the logarithmic functions of the materials distribution (34), cf. Fig. 10; for the linear functions (30), cf. Fig. 6; for the square functions (31), cf. Fig. 7; for the exponential functions (33), cf. Fig. 9; for the cubic functions (32), cf. Fig. 8. But for $k^{\prime \prime}>k^{\prime}$ (e.g. $k^{\prime \prime} / k^{\prime}=2$; 16) the functions are established in reverse order, i.e.: for the cubic functions of the materials distribution (32), cf. Fig. 8; for the exponential functions (33), cf. Fig. 9; for the square functions (31), cf. Fig. 7; for the linear functions (30), cf. Fig. 6; for the logarithmic functions (34), cf. Fig. 10.

\section{Remarks}

The modified tolerance averaging technique, presented and discussed in the book edited by Woźniak Cz., Michalak and Jędrysiak [29], has been applied to the heat conduction equation for transversally graded laminates (TGL). In this way, the governing equations of the tolerance model of those laminates have been obtained.

Summing up, we can formulate some general remarks.

- Using the tolerance averaging technique we have replaced the heat conduction differential equation (for transversally graded laminates (TGL)) with highlyoscillating, non-continuous coefficients by the system of differential equations with smooth, slowlyvarying coefficients.

- Derived equations of the proposed tolerance model take into account the effect of the microstructure size (the lamina thickness).

- For the stationary heat conduction we obtain exact analytical solutions to the aforementioned equations.

Analysing results of the example of the stationary heat conduction the following remarks can be formulated.

- Solutions to the governing equations of the tolerance model of the heat conduction take into account the microstructure of the TGL layer, cf. Fig. 5.

- Distributions of the temperature depend on:

- the functions describing the distribution of material properties in the TGL layer (i.e. the fraction ratios of materials),
- differences between heat conduction coefficients $k^{\prime}, k^{\prime \prime}$.

- The temperature decreases more strongly as the ratio of heat conduction coefficients $k^{\prime \prime} / k^{\prime}$ decreases.

- It can be observed that results obtained from the tolerance model are very close to results from the higher-order theory.

It seems that using the tolerance model other problems related to a heat conduction in TGL layers, e.g. non-stationary problems, inverted problems, can be considered.

Acknowledgements This contribution is supported by the Ministry of Science and Higher Education of Poland under grant No. N N506 398535.

Open Access This article is distributed under the terms of the Creative Commons Attribution Noncommercial License which permits any noncommercial use, distribution, and reproduction in any medium, provided the original author(s) and source are credited.

\section{References}

1. Aboudi J, Pindera M-J, Arnold SM (1999) Higher-order theory for functionally graded materials. Composites, Part B 30:777-832

2. Bansal Y, Pindera M-J (2003) Efficients reformulation of the thermoelastic higher-order theory for functionally graded materials. J Therm Stresses 26:1055-1092

3. Chiba R (2009) Stochastic thermal stresses in an FGM annular disc of variable thickness with spatially random heat transfer coefficients. Meccanica 44:159-176

4. Ferreira AJM, Batra RC, Roque, CMC, Qian, LF, Jorge, RMN (2006) Natural frequencies of functionally graded plates by a meshless method. Comp Struct 75:593-600

5. Goldberg RK, Hopkins DA (1995) Thermal analysis of a functionally graded material subject to a thermal gradient using the boundary element method. Comp Eng 5:793-806

6. Ignaczak J, Baczyński ZF (1997) On a refined heatconduction theory of micro-periodic layered solids. J Therm Stresses 20:749-771

7. Itoh Y, Takahashi M, Takano H (1996) Design of tungsten/copper graded composite for high heat flux components. Fusion Eng Des 31:279-289

8. Jędrysiak $\mathbf{J}$ (2003) The length-scale effect in the buckling of thin periodic plates resting on a periodic Winkler foundation. Meccanica 38:435-451

9. Jędrysiak J, Michalak B (2010) On the modelling of stability problems for thin plates with functionally graded structure. Thin-Walled Struct. doi:10.1016/j.tws.2010.09.005

10. Jędrysiak J, Radzikowska A (2007) On the modelling of heat conduction in a non-periodically laminated layer. $\mathrm{J}$ Theor Appl Mech 45:239-257

11. Jędrysiak J, Woźniak Cz (2006) On the propagation of elastic waves in a multiperiodically reinforced medium. Meccanica 41:553-569 
12. Jikov VV, Kozlov, SM, Oleinik, OA (1994) Homogenization of differential operators and integral functionals. Springer, Berlin

13. Kantor BY, Smetankina NV, Shupikov AN (2001) Analysis of non-stationary temperature fields in laminated strips and plates. Int J Solids Struct 38:8673-8684

14. Ke L-L, Yang J, Kitipornchai S (2009) An analytical study on the nonlinear vibration of functionally graded beams. Meccanica 45:743-752

15. Kim K-S, Noda N (2001) Green's function approach to three-dimensional heat conduction equation of functionally graded materials. J Therm Stresses 24:457-477

16. Kim K-S, Noda N (2002) A Green's function approach to the deflection of a FGM plate under transient thermal loading. Arch Appl Mech 72:127-137

17. Matysiak SJ (1991) On certain problems of heat conduction in periodic composites. Z Angew Math Mech 71:524-528

18. Michalak B, Woźniak Cz, Woźniak M (2007) Modelling and analysis of certain functionally graded heat conductors. Arch Appl Mech 77:823-834

19. Ootao Y, Tanigawa Y (2004) Transient thermoelastic problem of functionally graded thick strip due to nonuniform heat supply. Comp Struct 63:139-146

20. Ootao Y, Tanigawa Y (2006) Transient thermoelastic analysis for a functionally graded hollow cylinder. $\mathrm{J}$ Therm Stresses 29:1031-1046

21. Reiter T, Dvorak GJ, Tvergaard V (1997) Micromechanical models for graded composite materials. J Mech Phys Solids 45:1281-1302

22. Roque CMC, Ferreira AJM, Jorge RMN (2007) A radial basis function approach for the free vibration analysis of functionally graded plates using a refined theory. J Sound Vibr 300:1048-1070
23. Sadowski T, Ataya S, Nakonieczny K (2009) Thermal analysis of layered FGM cylindrical plates subjected to sudden cooling process at one side. Comparison of two applied methods for problem solution. Comput Mater Sci 45:624-632

24. Santos H, Mota Soares CM, Mota Soares CA, Reddy JN (2008) A semi-analytical finite element model for the analysis of cylindrical shells made of functionally graded materials under thermal shock. Comp Struct 86:10-21

25. Schmauder S, Weber U (2001) Modelling of functionally graded materials by numerical homogenization. Arch Appl Mech 71:182-192

26. Sladek J, Sladek V, Zhang Ch (2003) Transient heat conduction analysis in functionally graded materials by the meshless local boundary integral equation method. Comput Mater Sci 28:494-504

27. Suresh S, Mortensen A (1998) Fundamentals of functionally graded materials. The University Press, Cambridge

28. Tornabene F, Viola E (2009) Free vibration analysis of functionally graded panels and shells of revolution. Meccanica 44:255-281

29. Woźniak Cz, Michalak B, Jędrysiak J (eds) (2008) Thermomechanics of microheterogeneous solids and structures. Tolerance averaging approach. Wydawnictwo Politechniki Łódzkiej, Łódź

30. Woźniak Cz, Wierzbicki E (2000) Averaging techniques in thermomechanics of composite solids. Wydawnictwo Politechniki Częstochowskiej, Częstochowa

31. Woźniak M, Wierzbicki E, Woźniak Cz (2001) A macroscopic model of the diffusion and heat transfer processes in a periodically micro-stratified solid layer. Acta Mech 157:175-185 